\title{
Torsionswirkungen der Schuhsohlenrolle
}

\section{eine biomechanische Betrachtung}

\section{J. Grifka}

Orthopädische Klinik am ev. Fachkrankenhaus Ratingen (Chefarztgemeinschaft: Prof. Dr. O. Oest - Dr. F. Süssenbach)

Bei schmerzhaften Bewegungseinschränkungen im Mittelund Vorfußbereich kann mit einer fachgerecht angebrachten Schuhsohlenrolle auf einfache Art Erleichterung geschaffen werden. Doch auch, wenn die Abgrenzung zwischen Mittelfuß-, Ballen- und Zehenrolle regelrecht vorgenommen wurde, können durch einen falschen Verlauf der Rollenscheitellinie aufgrund der biomechanischen Auswirkungen ungünstige Torsionswirkungen resultieren, durch die die Schrittabwicklung erschwert und ein unphysiologisches Gangbild hervorgerufen wird. Um dies zu verhindern, ist es Aufgabe des Orthopäden, die verordnete Zurichtung auf die Richtigkeit der Ausführung zu überprüfen.

\section{Physiologische Schrittabwicklung}

Als Grundlage für die Rollenanbringung sei die physiologische Schrittabwicklung summarisch rekapituliert, wie diese in den Studien von S. Weil, U. H. Weil, 1966, und $H$. Hlavac, 1977, aufgezeigt und durch eigene Untersuchungen auf die Rollenwirkung übertragen wurde. Wenngleich das Gangbild von vielen Faktoren abhängig ist, wie z. B. Schrittlänge, Geh- oder Laufgeschwindigkeit, Alter, Körpergewicht und Muskelkraft, so läßt es sich doch auf allgemein zutreffende Grundelemente reduzieren. Bei der Einteilung in Schwungbeinphase und Stemmbeinphase wird letztere in drei Abwicklungsphasen unterteilt:

Auftrittsphase: Erster Bodenkontakt der Ferse in Supination. Durch Bewegungen in den Sprunggelenken findet der Fuß planen Bodenkontakt.

Aufliegephase: Der Fuß ist der Unterlage angepaßt und bietet die nötige Standsicherheit, damit die Resultierende aus Schwerkraft und Beschleunigung den Fuß von der Ferse zur Zehenspitze durchwandern kann (Abb. 1). Die Abwicklungslinie verläuft entsprechend der Gewölbekonstruktion von der Ferse über den belastungstragenden Fußaußenrand zum Vorfußbereich, bevor die Umlagerung in Pronation und die Hauptbelastung des ersten Metatarsalköpfchens erfolgt.

Abstoßphase: Mit dem Abheben der Ferse wird die Belastung im Vorfußbereich vermehrt; bei passiver Dorsalextension in den Zehengrundgelenken geschieht nun die Schrittabwicklung in Pronation des Vorfußes über die Großzehe, während die übrigen Zehen als stabilisierende Ausleger fungieren.

Diese Abfolge der Schrittabwicklung spiegelt sich an der Schuhsohlenabnutzung wider und steht mit der Belastungsverteilung unter der Fußsohle im Stand in Einklang (Abb. 1).

\section{Zusammenfassung}

Orientiert an der physiologischen Schrittabwicklung werden Forderungen an die Lage des Rollenscheitels bei Schuhsohlenrollen abgeleitet, um eine funktionsgerechte Schrittabwicklung entsprechend des zu versorgenden Krankheitsbildes sicherzustellen.

Durch nicht sachgerechte Rollenanbringung verursachte ungünstige Torsionswirkungen bedürfen wegen ihrer negativen Auswirkungen auf das Gangbild besonderer Berücksichtigung.

\section{Torsion Effects of Rolls in Shoe-Soles}

Reqirements concerning the location of the top of shoesole rolls are formulated on the basis of the physiological sequence of movements in the step, the aim being to ensure a functionally correct sequence of movements, corresponding to the condition to be treated.

Special attention should be paid to adverse torsion effects caused by inappropriate positioning of the rolls, since the torsion has negative effect on the gait pattern.

\section{Auswirkungen einer Schuhsohlenrolle}

Durch Anbringen einer Rolle unter der Schuhsohle mit entsprechendem Absatzangleich zur Wahrung der Laufebene bleibt die Auftrittsphase mit dem Aufsetzen der Ferse in der physiologischen Supinationsstellung unverändert. Bei dem sodann folgenden Einstellen des planen Bodenkontaktes durch die Bewegungen in den Sprunggelenken werden die Aufliegepunkte der Schuhfläche durch den Absatz und die Rolle, exakt den Rollenscheitel, bestimmt. Während ohne Rolle die Fläche von Ferse bis zu Mittelfußköpfchen I und $\mathrm{V}$ die Auftrittsfläche darstellt, ist nunmehr die distale Begrenzung durch die Rollenscheitellinie definiert (Abb. 2). Entsprechend ist die Standfläche und damit die Standsicherheit bei der Mittelfußrolle erheblich vermindert und bei der Zehenrolle wesentlich vergrößert (Abb. 3). Durchläuft nun in der Aufliegephase die Resultierende aus Schwerkraft und Beschleunigung die Fußsohle, so erfolgt bei Überschreiten der Rollenscheitellinie die gewünschte Abwicklung über die Rolle, die nun je nach Anbringen der Rolle als Ersatzbewegung für teilweise ruhiggestellte Fußgelenke fungiert bzw. eine rückhebelnde Wirkung ausübt.

\section{Torsionswirkungen einer Schuhsohlenrolle}

Von äußerst wichtiger Bedeutung ist der Rollenscheitellinienverlauf von Mittelfuß- und Ballenrolle, da der Fuß, wie gezeigt, durch die Abrollung über die aufliegende Scheitellinie einer Sohlenrolle in die Ausgangsposition für die letzte Phase der Schrittabwicklung gebracht wird. Verläuft der Rollenscheitel, wie in den meisten Ausführungen zu beobachten, von medial-distal nach lateral-proximal, so zeigt die Abwicklungslinie zum Außenrand des Vorfußes (Abb. 4). Durch die Abrollung über die Rolle wird also die Hauptbelastung auf den lateralen Vorfuß verlagert und die durch die Rolle bestimmte Bewegungsrichtung zeigt nach außen! Gleichzeitig wird der Vorfuß durch die an der Innenseite vorgezogene Rolle in Supination gebracht und somit die 

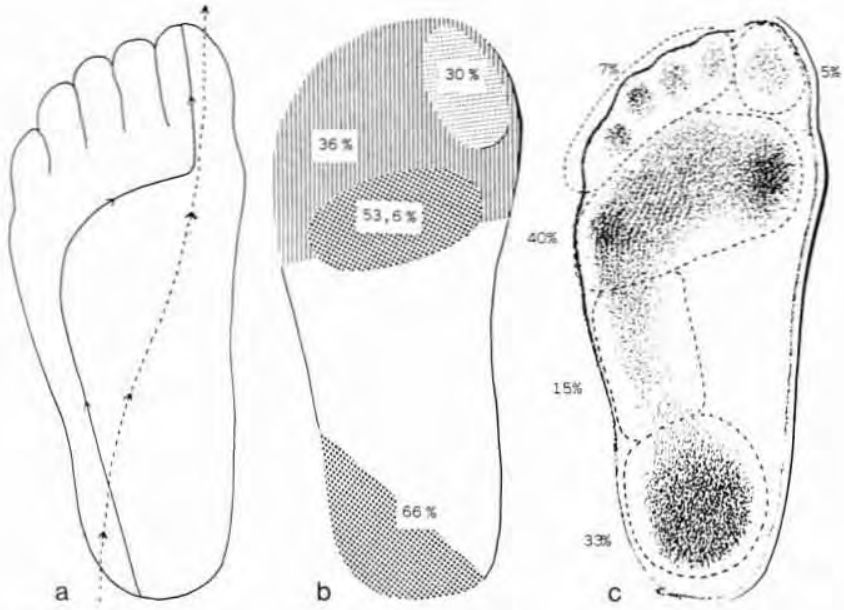

Abb. 1 a Verlauf von Abwicklungslinie $\rightarrow$ und Schwerelinie $\rightarrow$ unter der Fußsohle bei normalem Gehen (n. Hlavac, 1977)

Abb. 1b Schuhsohlenabnutzung mit Angabe der Abnutzungshäufigkeit in Prozent

Abb. 1c Belastungsverteilung unter der FuBsohle im Barfußstand in Prozent (aus Regenspurger, 1981)
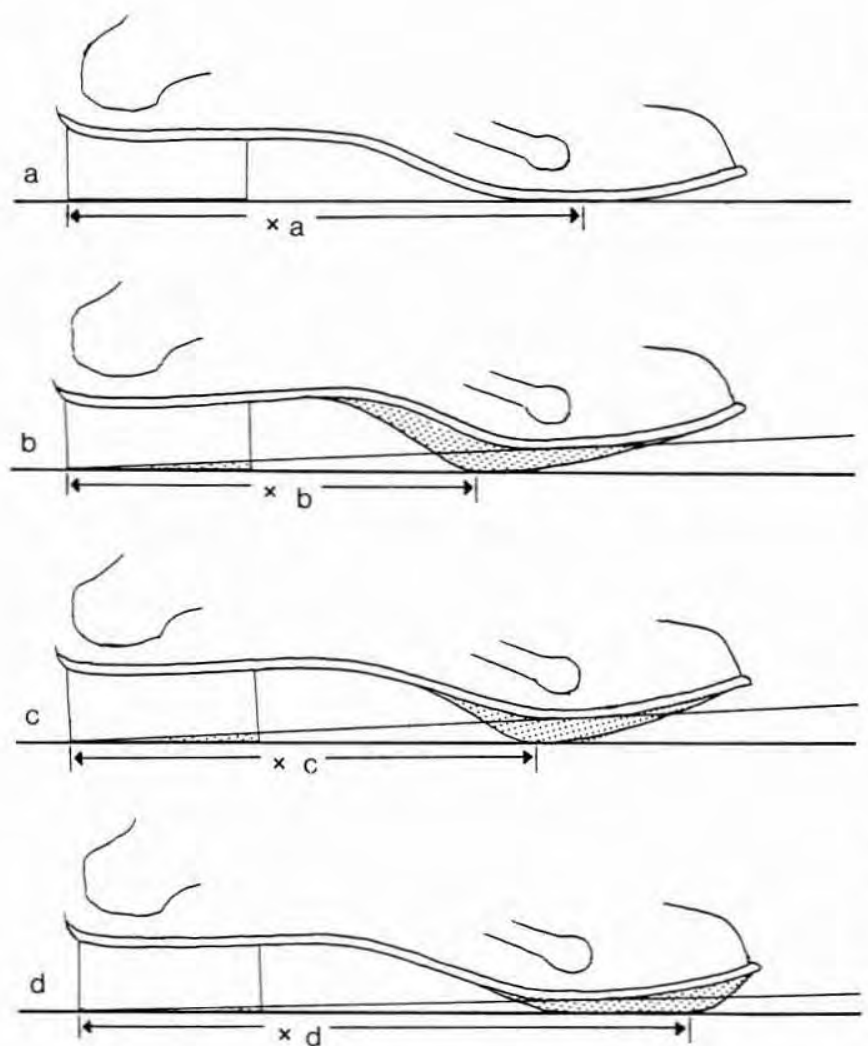

Abb. 3 Vergleichende Darstellung der Länge der Standflächen $(x)$ a) ohne Schuhzurichtung - übliche Länge der Standfläche b) bei Mittelfußrolle - erhebliche Verkürzung der Standflächenlänge, folglich Verminderung der Standsicherheit

c) bei Ballenrolle - Standfläche länger als bei Mittelfußrolle, aber kürzer als ohne Rolle

d) bei Zehenrolle - Verlängerung der Standflächenlänge, folglich Vergrößerung der Standsicherheit und Effekt der rückhebelnden Wirkung
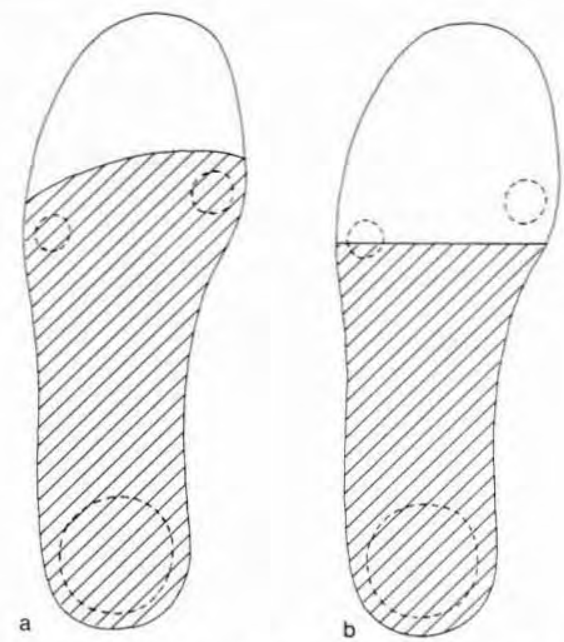

Abb. 2 Gegenüberstellung der Standflächen (schraffiert) ohne Schuhsohlenrolle (a) und mit Schuhsohlenrolle (b), deren Rollenscheitellinie die distale Begrenzung der Standfläche bildet; Auftrittspunkte von Mittelfußköpfchen I und V sowie Ferse gestrichelt dargestellt.

physiologische Umlagerung in Pronationsstellung zum Abstoßen über die mediale Vorfußpartie verhindert.

Schon durch die einzelnen Effekte, erst recht durch deren Summation, wird die physiologische Umlagerung im Vorfußbereich von Supination zur Pronation erschwert oder gar verhindert. In der Abstoßphase ist kein regelrechtes Abstoßen möglich, so daß die Schrittabwicklung behindert und das Gangbild gestört ist. Die Abstoßkraft ist vermindert. Die Rolle wird zum Hindernis.

Es genügt also nicht, lediglich die richtige Indikaiton zur Rollenversorgung zu stellen, sondern die schuhtechnische Ausführung muß auf ihre Richtigkeit überprüft werden, damit nicht eine fehlerhafte Rollenanbringung gar negative Auswirkungen hat.

\section{Richtige Rollenanbringung}

Aus den dargelegten Ausführungen über die physiologische Schrittabwicklung und die Funktion einer Schuhsohlenrolle folgt die fachgerechte Rollenanbringung (Abb. 4): Unter Berücksichtigung der individuellen, physiologischen Abduktionsstellung des Fußes, im wesentlichen durch die Außenrotation der Tibia bestimmt, wird die Scheitellinie der Rolle in 90 Grad zur Bewegungsrichtung gewählt und verläuft somit von medial-proximal nach lateral-distal unter der Schuhsohle. Durch das Vorziehen am SchuhauBenrand nach distal wird die Standsicherheit aufgrund der Hauptbelastung auf dem Fußaußenrand günstig beeinflußt. Die Abwicklungslinie verläuft ungestört über die belastungstragenden Anteile am Fußaußenrand. Über den Rollenscheitel wird in Bewegungsrichtung abgerollt ohne zusätzliche Ablenkung des Vorfußes aus der Bewegungsrichtung nach außen. Über die Scheitellinie erfolgt die Abrollung zur physiologischen Pronationsstellung des Vorfußes mit regelrechter Belastung des ersten Metatarsalköpfchens und der Großzehe und der Fuß kann ungehindert mit 

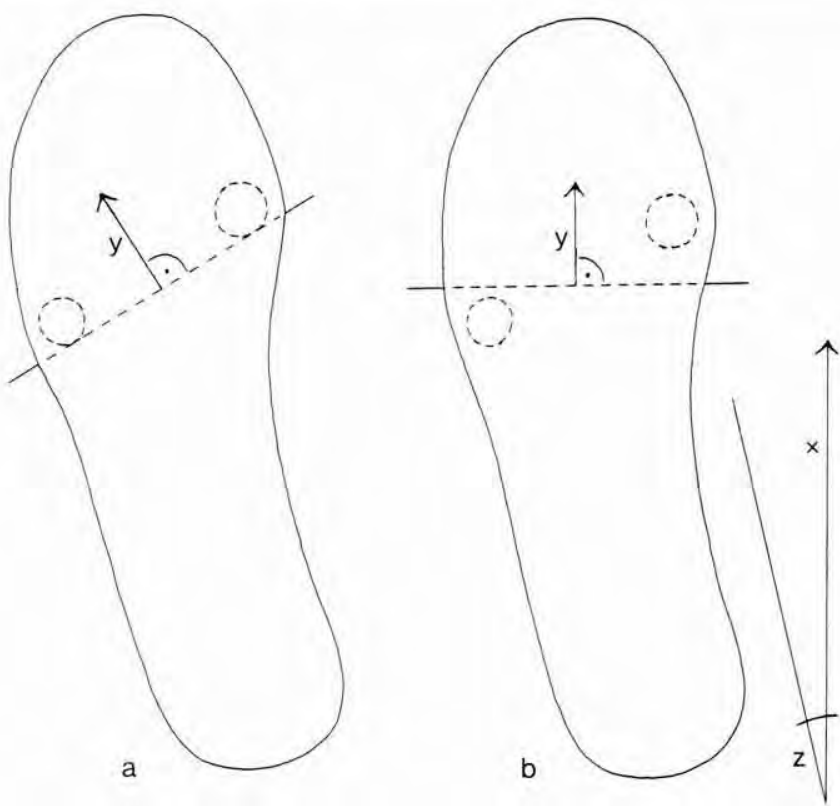

Abb. 4 Bei der Abwicklung über die Sohlenrolle wird durch den Verlauf der Rollenscheitellinie die Abwicklungsrichtung (y) im rechten Winkel zur Scheitellinie beeinflußt:

a) bei Verlauf der Scheitellinie von lateral-proximal nach medial-distal wird der Vorfuß in Supination gebracht und die Abwicklungsrichtung (y) nach außen abgelenkt

b) bei Beachtung des physiologischen Außenrotationswinkels ( $z$ ) und entsprechender Anbringung der Rollenscheitellinie im rechten Winkel zur Bewegungsrichtung $(x)$ (Scheitellinie verläuft von lateral-distal nach medial-proximal) kann in Bewegungsrichtung zur Pronationsstellung des Vorfußes abgewickelt werden; Abwicklungsrichtung (y) entspricht der Bewegungsrichtung $(\mathrm{x})$.

normaler muskulärer Abstoßkraft vom Boden abgestoßen werden.

\section{Ausnahme: Hallux Rigidus Rolle}

Bei der Hallux rigidus Rolle mit Hohllegung der Großzehe ist als Ausnahme eine Scheitellinienanbringung von medialdistal nach lateral-proximal indiziert (Abb. 5), um damit durch die Abrollung über den lateralen Vorfußbereich, Ablenken der Abstoßrichtung und konsekutiver Kraftminderung der Abstoßbewegung eine zusätzliche Druckentlastung unter der Großzehe und eine Bewegungsreduktion im Großzehengrundgelenk zu bewirken.

\section{Konsequenz}

Die Überlegungen zeigen, wie wichtig die fachgerechte Rollenanbringung ist und wie schon eine Änderung des Schei-

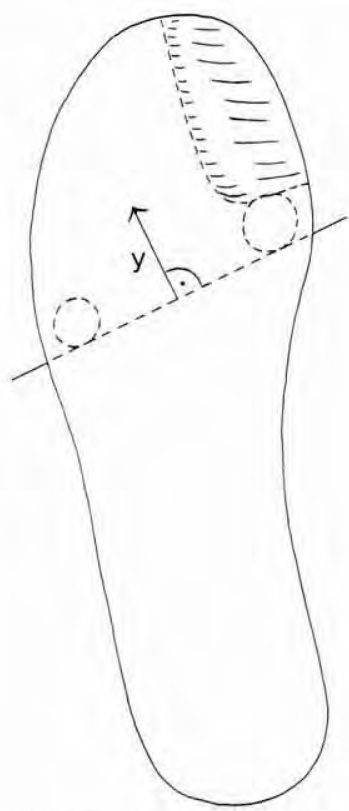

Abb. 5 Hallux rigidus Rolle. Vertiefung unter der Großzehe grob schraffiert dargestellt. Gewollte Ablenkung der Abwicklungsrichtung (y) auf den lateralen Vorfußbereich durch Anbringen der Rollenscheitellinie von lateral-proximal nach medial-distal.

tellinienverlaufs einer Schuhsohlenrolle gravierende Auswirkungen auf die Schrittabwicklung hat. Wegen der allzu häufigen fehlerhaften technischen Ausführung hat der verordnende Orthopäde die Kontrolle mit erhöhter Wachsamkeit durchzuführen.

\section{Literatur}

Hlavac, H.: The Foot Book. World Publications, Inc. Mountain View 1977

Regenspurger, G.: Spezielle Pathologie für Orthopädieschuhmacher. VEB

Verlag Volk und Gesundheit, Berlin 1981

Weil, S., U. H. Weil: Mechanik des Gehens. Georg Thieme Verlag, Stuttgart 1966

Dr. med. J. Grifka

Orthopädische Klinik am ev. Fachkrankenhaus

Rosenstr. 2

D-4030 Ratingen 1 